\title{
Mitochondrial function and aerobic capacity assessed by high resolution respirometry in Thoroughbred horses
}

\author{
D. Serteyn ${ }^{1,2,3^{*}}$, J. Ceusters ${ }^{1}$, S. Nonnenmacher ${ }^{2}$, K. Kirsch ${ }^{2,4}$, A. Mouithys-Mickalad ${ }^{1}$, T. Franck ${ }^{1,3}$, J.P. Lejeune ${ }^{3}$ and C. Sandersen ${ }^{1,2}$ \\ ${ }^{1}$ Centre for Oxygen Research and Development - CORD, University of Liege, Allée du VI août, 4000 Sart Tilman, Belgium; ${ }^{2}$ Clinical Department \\ of Companion Animals and Horses, Faculty of Veterinary Medicine, University of Liège, Boulevard de Colonster B41, 4000 Sart Tilman, \\ Belgium; ${ }^{3}$ Mont-le-Soie Equine Research Center, Mont-le-Soie 1, 6695 Vielsalm, Belgium; ${ }^{4}$ German Equestrian Olympic Committee, Freiherr- \\ von-Langen-Str. 15, 48231 Warendorf, Germany; didier.serteyn@ulg.ac.be
}

Received: 18 October 2015 / Accepted: 14 June 2016

(c) 2016 Wageningen Academic Publishers

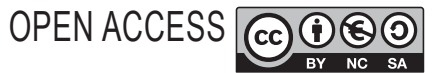

RESEARCH ARTICLE

\begin{abstract}
During the initial stages of training of young Thoroughbred horses, low intensity exercise is employed to increase aerobic capacity. High Resolution Respirometry (HRR) allows the determination of aerobic capacities in small samples of permeabilised muscle fibres. The aim of the study was to measure the mitochondrial function by HRR in Thoroughbred horses, to compare these values to Warmblood horses and to evaluate the effect of a 10-weeks training period. The mitochondrial function was measured by HRR using different substrate-uncoupler protocols (SUIT 1 and 2) in muscle microbiopsies from two groups of untrained horses: 17 Warmblood and 8 Thoroughbred and in the group of 8 Thoroughbred horses before and after a 10-week training period. The SUIT1 protocol employed to compare the two groups of horses showed that in Thoroughbred horses, the mean values for oxygen flux expressed as tissue mass-specific respiration were significantly higher for complex I (CI) Glutamate+Malate, $_{\text {, }}+$ complex II, and maximum electron transport capacities (ETSmax) than the mean values measured in Warmblood horses. The SUIT 1 and SUIT 2 protocols revealed large differences among Thoroughbred horses before and after training. The SUIT 2 protocols showed a significant difference for the complex I activity before and after training but only when the oxygen flux was expressed as percentage of ETSmax. This study shows the interest of HRR in equine sport medicine and exercise physiology, but shows that the technique requires further refinement. Indeed significant differences have been shown between the Thoroughbred and the Warmblood horses highlighting the need to have baseline data for each breed. The Thoroughbred horses had globally a high oxidative phosphorylation capacity with an increase of $\mathrm{CI}$ activity induced by an aerobic training program.
\end{abstract}

Keywords: equine exercise physiology, mitochondrion, oxygraphy, oxidative phosphorylation, training

\section{Introduction}

Physical training changes skeletal muscle anatomical structure and modifies its physiological and metabolic functions. Training is crucial to the improvement of performance and should be conducted in a way to increase fitness and to reduce the risk of injury (Hinchcliff and Geor, 2008; Lopez-Rivero et al., 1991, 1992; Rivero et al., 1995; Serrano et al., 2000). During the initial stages of training of young Thoroughbred horses, low intensity exercise, including trot and slow canter, is employed to increase aerobic capacity while slowly increasing skeletal loading. A number of studies have described the changes that occur in the aerobic capacity of horses when they undergo a similar training program (Evans and Rose, 1988; Hiraga et al., 1995, 1997). Recent works suggest that young racehorses might be able to achieve higher aerobic fitness during training without subjecting their musculoskeletal systems to increased loading and thus risking the development of lameness (Ohmura et al., 2013; Ringmark et al., 2015). One pronounced intramuscular adaptive response to aerobic exercise training is mitochondrial biogenesis, which increases the number of mitochondria and their oxidative capacity as well as modifying the mitochondrial mRNA 
expression and protein content (Eivers et al., 2010; Hawley et al., 2013; Irrcher et al., 2003).

Mitochondria are the most effective energy-generating organelles when oxygen is available. They produce energy through the oxidative phosphorylation (OXPHOS) process which couples oxygen consumption with the production of ATP. High-resolution respirometry (HRR) allows the determination of OXPHOS and electron transport capacities (ETS) in small samples (2 mg) of permeabilised muscle tissue (Gnaiger et al., 2005). Multiple Substrate-UncouplerInhibitor Titration (SUIT) protocols have been developed to screen the mitochondrial function of equine muscles. Two reference protocols (SUIT1 and SUIT2) were established for the investigation of mitochondrial function in equine skeletal muscle. They differ by the addition of pyruvate, the product of glycolysis thus studying mitochondrial respiration with (SUIT 2) or without (SUIT 1) assessment of aerobic glycolysis through the activity of the pyruvate dehydrogenase complex (Votion et al., 2012).

HRR has been used to study OXPHOS capacity for the evaluation of training programs in sport horses. Significant differences in mitochondrial respiratory function were observed between overweight, trained and untrained Warmblood horses (Votion et al., 2012). Previously, a significant increase of the complex-I (CI) activity after 10 weeks of training was observed in response to training in endurance horses (Votion et al., 2010). White et al. (2015) used HRR to identify changes in mitochondrial function induced by submaximal training of young Quarter horses. To our knowledge, the HRR has not been used to assess OXPHOS and ETS capacities in Thoroughbred horses. We hypothesise that mitochondrial function (measured using HHR) will differ when Thoroughbreds are compared to Warmbloods. Secondarily, we hypothesise that 10 weeks of training will alter mitochondrial function in Thoroughbreds.

\section{Material and methods}

\section{Horses}

All procedures of this study were approved by the Animal Ethic Commission of the University of Liege (agreement no. 629). The comparison between the mitochondrial function of Warmblood and Thoroughbred horses was studied in two different groups of horses. The first group consisted of 17 healthy adult Warmblood horses (12 females and 5 males). They had a mean age of $8 \pm 3$ years. They were untrained and had a body condition score ranging from 2 to 3 out of 5 . The second group consisted of 8 young ( 4 males and 4 females) Thoroughbred race horses with a mean age of $3 \pm 0.6$ years. This group was sampled twice, once before the training period ( $\mathrm{T} 0$ ), and once after the training period (T10). All Thoroughbred horses had a rest period of three months before entering the training protocol. This training period lasted for 10 weeks and was controlled by the trainer of the horses. The daily work consisted of a trotting warmup period ( $20 \mathrm{~min}, 220 \mathrm{~m} / \mathrm{min}$ ) in an indoor arena followed by a walk period $(5 \mathrm{~min}$ ) to reach the training track. Two galloping periods $(10 \mathrm{~min})$ were included on each hand at a speed of $380-420 \mathrm{~m} / \mathrm{min}$ with $5 \mathrm{~min}$ at walk between the two periods. After the gallop, the horses returned to the indoor arena for a last trotting $(10 \mathrm{~min})$. Furthermore, each Thoroughbred horse was placed in the horse walker for $30 \mathrm{~min}$ then let loose in individual paddock for $60 \mathrm{~min}$.

\section{Muscle micro-biopsy}

Approximately $20 \mathrm{mg}$ of muscle were collected from the triceps brachii muscle using a $14 \mathrm{G}$ semi-automatic biopsy needle (Temno Evolution, Carefusion, Chateaubriant, France). Briefly, the sampling site was shaved and desensitised by subcutaneous injection of $0.5 \mathrm{ml}$ of lidocaine (Xylocaine 2\%, AstraZeneca, Brussels, Belgium), and aseptically prepared. Muscle microbiopsy specimens were taken at $40 \mathrm{~mm}$ depth in the long head of the triceps brachii muscle through a skin incision. Two to three muscle samples were obtained via the same skin opening and transferred immediately into ice-cold relaxing solution BIOPS containing $10 \mathrm{mM} \mathrm{CaK}_{2}$-EGTA, $7.23 \mathrm{mM} \mathrm{K}_{2}$-EGTA, $20 \mathrm{mM}$ imidazole, $20 \mathrm{mM}$ taurine, $50 \mathrm{mM}$ K-MES, $0.5 \mathrm{mM}$ dithiothreitol, $6.56 \mathrm{mM} \mathrm{MgCl}_{2}, 5.77 \mathrm{mM}$ ATP and $15 \mathrm{mM}$ phosphocreatine adjusted to $\mathrm{pH}$ 7.1. Samples were kept at $4{ }^{\circ} \mathrm{C}$ until further preparation.

\section{High resolution respirometry}

Connective tissue was removed and muscle fibres were mechanically separated using two pairs of forceps with sharp tips. Complete permeabilisation of the cell membrane was obtained by gentle agitation for $30 \mathrm{~min}$ at $4{ }^{\circ} \mathrm{C}$ in $2 \mathrm{ml}$ of BIOPS solution containing $50 \mu \mathrm{g} / \mathrm{ml}$ saponin. The fibre bundles were rinsed by agitation for $10 \mathrm{~min}$ in ice-cold mitochondrial respiration medium (MiR05; 0.5 mM EGTA, $3 \mathrm{mM} \mathrm{MgCl}_{2}$, $60 \mathrm{mM} \mathrm{K-lactobionate,} 20 \mathrm{mM}$ taurine, 10 $\mathrm{mM} \mathrm{KH} \mathrm{PO}_{4}, 20 \mathrm{mM}$ Hepes, $110 \mathrm{mM}$ sucrose and $1 \mathrm{~g} / \mathrm{l}$ bovine serum albumin essentially fatty acid free adjusted to $\mathrm{pH}$ 7.1). The permeabilised muscle fibres were immediately used for HRR.

One to $2.5 \mathrm{mg}$ (microbalance; Mettler Toledo, Zaventem, Belgium) of permeabilised muscle fibres were added to each Oxygraph-2k chamber (Oroboros Instruments, Innsbruck, Austria) containing $2 \mathrm{ml}$ of MiR05 at $37.0{ }^{\circ} \mathrm{C}$. Oxygen concentration in $\mu \mathrm{M}$, and oxygen flux per muscle mass ( $\mathrm{pmol} \mathrm{O}_{2} / \mathrm{s} \mathrm{mg}$ ) were recorded online using DatLab software (Oroboros Instruments). After calibration of the oxygen sensors with ambient air, a few ml of oxygen were introduced into the gas phase above the stirred aqueous phase in the partially closed chambers to reach a 
concentration of $500 \mu \mathrm{M} \mathrm{O}_{2}$. During the complete SUIT protocols (approximately $1 \mathrm{~h}$ ), the oxygen level in the chambers has to be maintained between 200 and $500 \mu \mathrm{M}$ $\mathrm{O}_{2}$ to avoid any oxygen limitation of respiration (Pesta and Gnaiger, 2012). Even though air-level oxygen pressure is not a limiting factor for respirometric studies with isolated mitochondria, low oxygen levels $\left(<200 \mu \mathrm{M} \mathrm{O}_{2}\right)$ have to be avoided in the present setting because of restricted oxygen diffusion in permabilised muscle fibres (Gnaiger, 2009). Such oxygen dependence was avoided in the present study by reoxygenating the oxygraphy chamber when the $\mathrm{O}_{2}$ level in the medium dropped towards $200 \mu \mathrm{M}$. As recommended, respiratory flux was corrected on-line for instrumental background, determined at experimental oxygen levels.

In the SUIT1 protocol, electron flow through CI was supported by the NADH linked substrates glutamate + malate $(\mathrm{G}+\mathrm{M} ; 10$ and $2 \mathrm{mM})$ with subsequent addition of ADP $(2.5 \mathrm{mM})$. In the SUIT2 protocol electron flow through CI was first supported by the NADH-linked substrates pyruvate + malate $(\mathrm{P}+\mathrm{M} ; 5$ and $2 \mathrm{mM})$ to assess pyruvate dehydrogenase activity (aerobic glycolysis) and then by adding the glutamate $(\mathrm{G})$ at $10 \mathrm{mM}$ with subsequent addition of ADP $(2.5 \mathrm{mM})$. ADP-stimulated respiration represents OXPHOS capacity. Then, we added succinate (S; $10 \mathrm{mM}$ ) for convergent electron flow through complex I and II (CI+CII), supported by $\mathrm{G}+\mathrm{M}+\mathrm{S}$ or $\mathrm{P}+\mathrm{M}+\mathrm{G}+\mathrm{S}$. The capacity of the phosphorylation system (adenine nucleotide translocase, inorganic phosphate transporter, and ATP synthase) may limit OXPHOS capacity with an apparent excess capacity of the ETS over the phosphorylation system (Gnaiger, 2009). This was tested by stepwise addition of the uncoupler carbonyl cyanide 4-(trifluoromethoxy)phenylhydrazone incrementally (FCCP) $(0.05 \mu \mathrm{M}$ followed by several additions of $0.025 \mu \mathrm{M}$ until maximal oxygen flux was reached), obtaining maximal ETS capacity (ETSmax), with convergent electron flow through CI+CII. Electron input into the Q-junction through complex II (CII) alone was subsequently induced by inhibition of $\mathrm{CI}$ by rotenone $(0.5 \mu \mathrm{M})$. Finally, residual oxygen consumption was obtained by addition of antimycin A $(2.5 \mu \mathrm{M})$ to block electron transfer through complex III (CIII). Oxygen fluxes were corrected by subtracting residual oxygen consumption from each measured mitochondrial steady-state.

Integrity of the outer mitochondrial membrane was tested by adding $10 \mu \mathrm{M}$ cytochrome $\mathrm{C}$ after ADP in the presence of substrates feeding electrons into CI. Injury of the outer mitochondrial membrane leads to loss of cytochrome $\mathrm{C}$ from the mitochondria, and to significant stimulation of respiration following addition of exogenous cytochrome C to the respiration medium (Kuznetsova et al., 2004).

Oxygen flux was expressed as tissue mass-specific respiration (per mg), or as flux control ratios (FCR), with internal normalisation for maximum ETS capacity. The
SUIT1 and SUIT2 protocols were used to compare the two breeds (Warmblood versus untrained Thoroughbred) and to study the effect of the training in the Thoroughbred horses.

\section{Statistical analysis}

Respirometry measurements were performed at least in duplicate for each protocol and the mean of the two closest values was used for further calculation. For both groups of horses (the Warmblood horses and the Thoroughbred horses before training), and for the Thoroughbred horses before and after training, all data are presented as mean values \pm SD. A Levene's test was performed to assume the equality of variances between each group of data. An independent $\mathrm{t}$-test was used to compare the mean oxygen flux values of $\mathrm{CI}, \mathrm{CI}+\mathrm{CII}$, ETSmax and CII alone between the Warmblood and the Thoroughbred horses before training. A paired samples t-test was used to compare the mean values of the same parameters before and after training. A value of $P<0.05$ was considered as level of significance (Medcalc, MedCalc Software, Version 12.7.7.; Ostend, Belgium).

\section{Results}

\section{Comparison between untrained Warmblood and Thoroughbred horses}

The SUIT1 protocol employed to compare the two groups of horses showed that in Thoroughbred horses, the mean values for oxygen flux expressed as tissue mass-specific respiration were significantly higher for $\mathrm{CI}_{G+M}, \mathrm{CI}+\mathrm{CII}$, and ETSmax than the mean values measured in Warmblood horses. Figure $1 \mathrm{~A}$ illustrates mean values $( \pm \mathrm{SD})$ obtained with the SUIT1 protocol. Table 1 displays the oxygen flux expressed as FCR. In the Warmblood horses, CI and CII alone activities were significantly higher.

Concerning the SUIT2 protocol, all the oxygen fluxes expressed as tissue mass-specific respiration were significantly higher in the Thoroughbred $t$ han in the Warmblood horses (Figure 1B). When the oxygen flux was expressed as FCR, the part of ETSmax used via $\mathrm{CI}_{P_{+} M}$ and $\mathrm{CI}_{P+M+G}$ and $\mathrm{CII}$ alone were significantly higher in the Warmblood horses (Table 2).

\section{Comparison between Thoroughbred horses before (TO) and after training (T1)}

The SUIT1 protocol did not show any significant differences between $\mathrm{T} 0$ and $\mathrm{T} 1 \mathrm{in}$ Thoroughbred horses for the mean values of oxygen flux expressed as tissue mass-specific respiration (Figure 2) or expressed as flux control ratio.

Concerning the SUIT2 protocol, the individual values expressed as tissue mass-specific respiration of each 
A

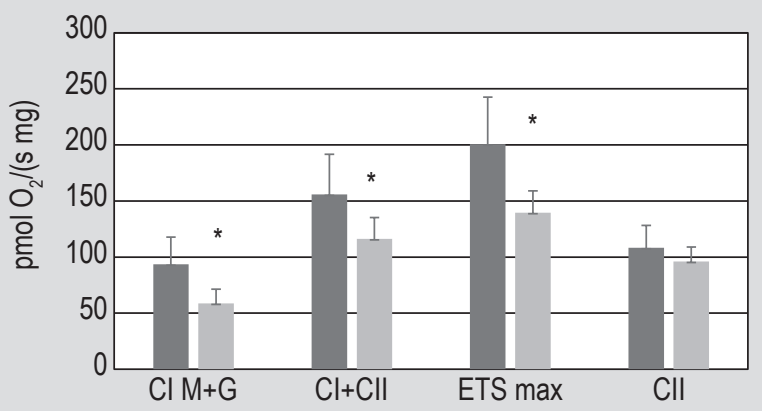

B

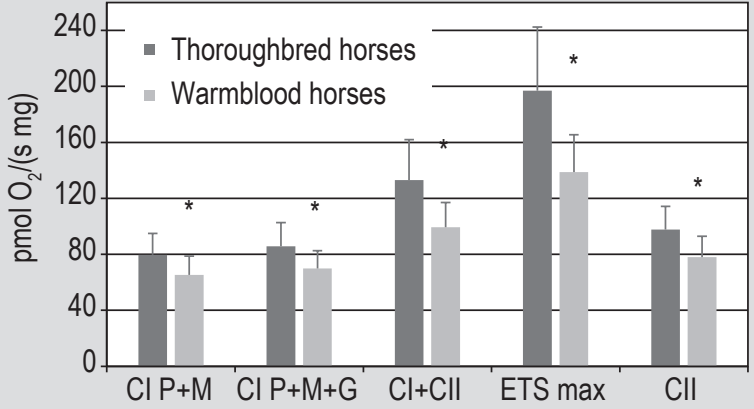

Figure 1. Tissue mass-specific respirations (mean \pm standard deviation of oxygen flux per $\mathrm{mg}$ of muscle) of Thoroughbred and Warmblood horses. (A) SUIT 1. (B) SUIT-2. $\mathrm{Cl}_{\mathrm{M}+\mathrm{G}}$ : oxygen flux via mitochondrial complex I in the presence of malate and glutamate; $\mathrm{Cl}_{\mathrm{P}+\mathrm{M}}$ : oxygen flux via mitochondrial complex I in the presence of pyruvate and malate; $\mathrm{Cl}_{\mathrm{P}+\mathrm{M}+\mathrm{G}}$ : oxygen flux via mitochondrial complex I in the presence of pyruvate, malate and glutamate; $\mathrm{Cl}+\mathrm{Cll}$ : oxygen flux via mitochondrial complex I and II; ETSmax: maximal respiratory capacity after total uncoupling with carbonyl cyanide 4-[trifluoromethoxy]phenylhydrazone; Cll: oxygen flux via mitochondrial complex II alone. * $P<0.05$ Thoroughbred vs Warmblood.

Table 1. Number, age and SUIT1 values expressed as flux control ratios (mean \pm standard deviation) of Thoroughbred and Warmblood horses. ${ }^{1}$

\begin{tabular}{|c|c|c|c|c|c|c|}
\hline Horse & $\mathrm{n}$ & Age (year) & $\mathrm{Cl}_{\mathrm{M}+\mathrm{G}}$ & $\mathrm{Cl}+\mathrm{Cll}$ & ETSmax ${ }^{2}$ & $\mathrm{Cll}$ \\
\hline Thoroughbred & 8 & $3 \pm 0.6$ & $46.2 \pm 5.5$ & $77.6 \pm 7.1$ & 100 & $54.6 \pm 6.9$ \\
\hline Warmblood & 17 & $8 \pm 3$ & $41.6 \pm 5.0$ & $83.4 \pm 9.4$ & 100 & $68.9 \pm 7.2$ \\
\hline$P$-value & & & 0.055 & 0.13 & & 0.001 \\
\hline
\end{tabular}

Table 2. Number, age, and SUIT2 values expressed as flux control ratios [FCR; mean \pm SD] of Thoroughbred and Warmblood horses. ${ }^{1}$

\begin{tabular}{lcllllll} 
Horse & $\mathbf{n}$ & Age (year) & $\mathrm{Cl}_{\mathrm{P}+\mathrm{M}}$ & $\mathrm{Cl}_{\mathrm{P}+\mathrm{M}+\mathrm{G}}$ & $\mathrm{Cl}+\mathrm{Cll}$ & $\mathrm{ETSmax}^{2}$ & $\mathrm{Cll}$ \\
& & & & & & & \\
Thoroughbred & 8 & $3 \pm 0.6$ & $39.7 \pm 4.6$ & $42.9 \pm 3.6$ & $71.4 \pm 3.6$ & 100 & $51.9 \pm 3.7$ \\
Warmblood & 17 & $8 \pm 3$ & $46.1 \pm 3.8$ & $49.6 \pm 4.4$ & $72.6 \pm 4.3$ & 100 & $55.4 \pm 3.6$ \\
$P$-value & & & 0.003 & 0.002 & 0.51 & & 0.04 \\
\hline
\end{tabular}

\footnotetext{
${ }^{1} \mathrm{ETSmax}=$ maximal respiratory capacity; $\mathrm{Cl}_{\mathrm{P}+\mathrm{M}+\mathrm{G}}=$ part of $\mathrm{ETSmax}$ used via mitochondrial complex I in the presence of pyruvate and malate; $\mathrm{Cl}_{\mathrm{P}+\mathrm{M}+\mathrm{G}}$ : part of ETSmax used via mitochondrial complex I in the presence of pyruvate, malate and glutamate; $\mathrm{Cl}+\mathrm{CII}=$ part of ETSmax used via mitochondrial complex I and II; CII = part of ETSmax used via mitochondrial complex II.

${ }^{2}$ Flux control ratios are standardised for the respective ETSmax of each group (ETSmax $=100 \%$ for each experimental group).
}

Thoroughbred horse before and after the training period are reported in Table 3. Large individual variations between the horses were identified. The comparison of the mean values before and after training did not show any significant differences. When the oxygen flux was expressed as FCR, with internal normalisation for maximum ETS capacity, a significant difference was observed after the training period for the CI activity compared to the values obtained before training. The results are illustrated in the Figure 3. 
Table 3. Tissue mass-specific respirations (oxygen flux per mg of muscle) observed for Thoroughbred horses before and after training using the SUIT2 protocol. ${ }^{1}$

\begin{tabular}{|c|c|c|c|c|c|c|c|c|c|c|}
\hline \multirow{2}{*}{$\begin{array}{l}\text { Thoroughbred } \\
\text { (pmol oxygen/ } \\
\text { s mg muscle) }\end{array}$} & \multicolumn{5}{|c|}{ Before training } & \multicolumn{5}{|c|}{ After training } \\
\hline & $\mathrm{Cl}_{\mathrm{P}+\mathrm{M}}$ & $\mathrm{Cl}_{\mathrm{P}+\mathrm{M}+\mathrm{G}}$ & $\mathrm{Cl}+\mathrm{Cll}$ & ETSmax & $\mathrm{Cll}$ & $\mathrm{Cl}_{\mathrm{P}+\mathrm{M}}$ & $\mathrm{Cl}_{\mathrm{P}+\mathrm{M}+\mathrm{G}}$ & $\mathrm{Cl}+\mathrm{Cll}$ & ETSmax & $\mathrm{Cll}$ \\
\hline Horse 1 & 81.76 & 90.586 & 144.49 & 210.59 & 109.91 & 69.02 & 74.63 & 111.85 & 158.18 & 88.899 \\
\hline Horse 2 & 52.067 & 54.818 & 112.41 & 149.57 & 89.25 & 98.97 & 101.55 & 163.22 & 264.59 & 118.765 \\
\hline Horse 3 & 102.93 & 138.786 & 235.44 & 313.001 & 152.80 & 73.45 & 77.17 & 127.041 & 184.65 & 91.58 \\
\hline Horse 4 & 82.41 & 86.30 & 144.049 & 212.81 & 112.72 & 79.26 & 88.85 & 138.84 & 180.24 & 92.705 \\
\hline Horse 5 & 97.68 & 98.96 & 160.054 & 243.72 & 117.37 & 88.76 & 96.004 & 149.902 & 229.283 & 106.72 \\
\hline Horse 6 & 100.61 & 101.72 & 160.97 & 219.48 & 114.21 & 56.85 & 58.809 & 80.69 & 123.508 & 69.224 \\
\hline Horse 7 & 85.809 & 90.02 & 132.51 & 186.49 & 91.30 & 71.23 & 77.203 & 123.91 & 197.404 & 94.508 \\
\hline Horse 8 & 74.567 & 79.84 & 135.15 & 181.86 & 95.67 & 100.11 & 111.59 & 169.013 & 237.103 & 118.89 \\
\hline Mean & 84.732 & 92.629 & 153.132 & 214.69 & 110.40 & 79.71 & 85.73 & 133.059 & 196.87 & 97.662 \\
\hline SD & 16.634 & 23.596 & 36.738 & 48.7926 & 20.341 & 15.201 & 17.02 & 28.87 & 45.607 & 16.63 \\
\hline
\end{tabular}

${ }^{1} \mathrm{Cl}_{\mathrm{P}+\mathrm{M}}=$ oxygen flux via mitochondrial complex I with pyruvate and malate as substrates; $\mathrm{Cl}_{\mathrm{P}+\mathrm{M}+\mathrm{G}}=$ oxygen flux via mitochondrial complex I with pyruvate, malate and glutamate as substrates; $\mathrm{Cl}+\| \mathrm{I}=$ oxygen flux via mitochondrial complex I and II; ETSmax = maximal respiratory capacity after total uncoupling with carbonyl cyanide 4-[trifluoromethoxy]phenylhydrazone; CII = oxygen flux via mitochondrial complex II alone.

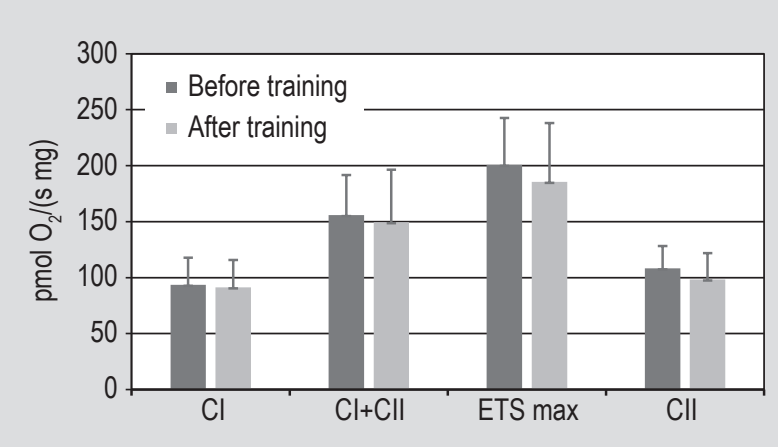

Figure 2. SUIT 1: tissue mass-specific respirations (mean \pm standard deviation of oxygen flux per mg of muscle) of Thoroughbred horses before and after training. Cl: oxygen flux via mitochondrial complex I in the presence of malate and glutamate; $\mathrm{Cl}+\mathrm{Cll}$ : oxygen flux via mitochondrial complex I and II; ETSmax: maximal respiratory capacity after total uncoupling with carbonyl cyanide 4-[trifluoromethoxy]phenylhydrazone; Cll: oxygen flux via mitochondrial complex II alone.

\section{Discussion}

High resolution respirometry is a validated technique for the assessment of mitochondrial function. SUIT protocols for the analysis of oxidative phosphorylation improve our understanding of mitochondrial respiratory control and the pathophysiology of mitochondrial diseases (Gnaiger, 2009; Pesta and Gnaiger, 2012). An early defect of oxidative phosphorylation in the failing human heart was demonstrated by HRR (Lemieux et al., 2011). In type 2 diabetes patients, Phielix et al. (2008) showed, by using

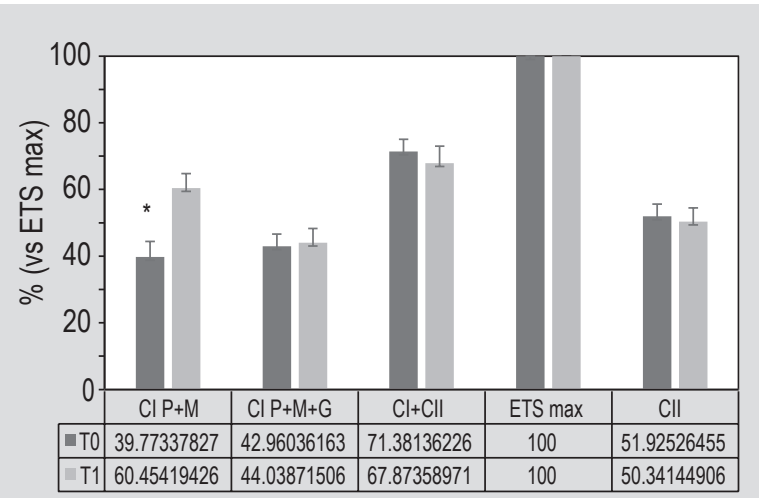

Figure 3. Flux control ratios (mean \pm standard deviation), standardised for their respective maximal respiratory capacities $(E T S \max =100 \%$ ), of Thoroughbred horses before (T0) and after (T1) training. $\mathrm{Cl}_{\mathrm{P}+\mathrm{M}}$ : part of ETSmax used via mitochondrial complex $\mathrm{I}$ in the presence of pyruvate and malate; $\mathrm{Cl}_{\mathrm{P}+\mathrm{M}+\mathrm{G}}$ : part of ETSmax used via mitochondrial complex I in the presence of pyruvate, malate and glutamate; $\mathrm{Cl}+\mathrm{Cll}$ : part of ETSmax used via mitochondrial complex I and II; ETSmax: maximal respiratory capacity; Cll: part of ETSmax used via mitochondrial complex II. ${ }^{*} P<0.05$ before vs after training.

multiple substrates, that compromised mitochondrial function resides at the level of the phosphorylation system. Increasing aerobic fitness in man is associated with an increase of mitochondrial quality and quantity (Jacobs and Lundby, 2013). Likewise in the horse, HRR has been used in exercise physiology as well as in pathology. Previous results obtained in horses demonstrated that endurance training 
induced a significant increase of ETS and oxygen fluxes (Votion et al., 2010, 2012). More recently, mitochondrial dysfunction was identified by HRR during laminitis (Serteyn et al., 2014) and exercise-induced rhabdomyolysis (Houben et al., 2015).

To our knowledge, the present study is the first to analyse muscle mitochondrial function in Thoroughbred race horses using HRR. A microbiopsy of only $20 \mathrm{mg}$ of muscle is largely sufficient to realise the SUIT protocols. Due to this minimal invasive approach, mitochondrial function can easily be investigated by HRR in sport and racing horses. The triceps brachii and the gluteus muscle can be used for HRR studies (Votion et al., 2012). In the present study the triceps brachii was chosen because sampling of the front limb appeared to be safer for the investigator than the propulsive muscles of the hind limb. However, in young quarter horses, White et al. (2015) used HRR to demonstrate that submaximal exercise training appeared to further enhance mitochondrial efficiency in the gluteus medius muscle.

As expected, our results (SUIT 1 and SUIT 2) comparing the oxygen flux expressed as tissue mass-specific respiration, showed that mitochondrial function was more efficient in Thoroughbred than in Warmblood horses. This is explained by the superior athletic ability attributable to the high maximal aerobic capacity, the ability to increase oxygen-carrying capacity through splenic contraction at the onset of exercise, its increased muscle bulk, large intramuscular stores of energy substrates (glycogen in particular), high mitochondrial volume in muscle, and the high proportion of fast-twitch muscle fibres (Rivero et al., 2007). However, when the oxygen flux are expressed as FCR (vs ETSmax), a different pattern between the two breeds was observed. With SUIT1, the Thoroughbred horses use a greater part of their maximal respiratory capacity for CI whereas the Warmblood ones use a greater part of it for the CII. Mitochondrial complex II (succinate dehydrogenase) oxidises succinate to fumarate and thereby creating a direct link between the citric acid cycle and the respiratory chain (Cecchini, 2003). The SUIT2 protocol shows that Warmblood horses need to use greater parts of their maximal respiratory capacity for CI and CII to maintain their energetic state.

In Thoroughbred horses, the effect of training on the oxygen flux expressed as tissue mass-specific respirations did not show any significant difference with the SUIT1 and the SUIT2 protocols. The intensity of the training program in this study was probably too low to induce a significant increase of the mitochondrial content in the muscle. Furthermore, a large individual variation was also observed and this was probably explained by the fact that the sampling sites were not exactly the same and the number of mitochondria differed between the microbiopsies.
Proliferation of mitochondria is sometimes associated with an increase in citrate synthase activity per cell, but its activity in a specific tissue is frequently constant when expressed per mitochondrial protein. To reduce the impact of this factor, some authors propose to express the tissue respiration data per citrate synthase activity (Renner et $a l ., 2003)$.

In this study, the oxygen flux was expressed as FCR, with internal normalisation for maximum ETS capacity. The SUIT 2 protocol, using pyruvate, demonstrated a significant difference after the training period for the CI activity compared to the values obtained before training. The fact that the training induced a significant increase of the $\mathrm{CI}$ activity in the presence of pyruvate indicates that the mitochondrial aerobic glycolysis pathway was improved. As the part used via $\mathrm{CI}$ in the presence of malate and glutamate alone is not affected, the 10 weeks of training have a significant effect on the pyruvate dehydrogenase activity. Exercise training has previously been shown to increase skeletal muscle protein content of pyruvate dehydrogenase in young human subjects (Leblanc et al., 2004).

This first study reporting HRR measurements in Thoroughbred had some limitations. Indeed, the mean ages of the two groups were different. Some information concerning the precise physical activity and nutrition of each horse were missing, especially for the Warmblood horses.

To conclude, this study shows the interest of HRR in equine sport medicine and exercise physiology. Significant differences have been shown between Thoroughbred and Warmblood horses highlighting the need to have baseline data for each breed. The Thoroughbred race horses had globally a high oxidative phosphorylation capacity with an increase of $\mathrm{CI}$ activity induced by an aerobic training program. For the future studies, the results showed that it is crucial and necessary to differentiate mitochondrial content from function.

\section{References}

Cecchini, G., 2003. Function and structure of complex II of the respiratory chain. Annual Review of Biochemistry 72: 77-109.

Eivers, S.S., McGivney, B.A., Fonseca, R.G., MacHugh, D.E., Menson, K., Park, S.D., Rivero, J.L., Taylor, C.T., Katz, L.M. and Hill, E.W., 2010. Alterations in oxidative gene expression in equine skeletal muscle following exercise and training. Physiology Genomics 40: 83-93.

Evans, D.L. and Rose, R.J., 1988. Cardiovascular and respiratory responses to submaximal exercise training in the Thoroughbred horse. European Journal of Physiology 411: 316-321.

Gnaiger, E., 2009. Capacity of oxidative phosphorylation in human skeletal muscle: new perspectives of mitochondrial physiology. International Journal of Biochemistry and Cell Biology 41: 1837-1845. 
Gnaiger, E., Wright-Paradis, C., Sondergaard, H., Lundby, C., Calbet, J.A., Saltin, B., Helge, J. and Boushel, R., 2005. High-resolution respirometry in small biopsies of human muscle: correlations with body mass index and age. Mitochondria Physiology Network 10: 14-15.

Hawley, J.A., Burke, L.M., Phillips, S.M. and Spriet, L.L., 2013. Optimizing intramuscular adaptations to aerobic exercise: effects of carbohydrate restriction and protein supplementation on mitochondrial biogenesis. Advances in Nutrition 4: 657-664.

Hinchcliff, K.W. and Geor, R.J., 2008. The horse as an athlete: a physiological overview. In: Hinchcliff, K.W., Geor, R.J. and Kaneps, A.J. (eds.) Equine exercise physiology, the science of exercise in the athletic horse. Elsevier, Philadelphia, PA, USA, pp. 2-11.

Hiraga, A., Kai, M., Kubo, K. and Erickson, B.K., 1995. The effect of long slow distance training on aerobic work capacity in young Thoroughbred horses. Journal of Equine Veterinary Science 6: 1-6.

Hiraga, A., Kai, M., Kubo, K. and Sugano, S., 1997. Effects of training intensity on cardiopulmonary function in 2 year-old Thoroughbred horses. Journal of Equine Veterinary Science 8: 75-80.

Houben, R., Leleu, C., Fraipont, A., Serteyn, D. and Votion, D.M., 2015. Determination of muscle mitochondrial respiratory capacity in Standardbred racehorses as an aid to predicting exertional rhabdomyolysis. Mitochondrion 24: 99-104.

Irrcher, I., Adhihetty, P.J., Joseph, A.M., Ljubicic, V. and Hood, D.A., 2003. Regulation of mitochondrial biogenesis in muscle by endurance exercise. Sports Medicine 33: 783-793.

Jacobs, R.A. and Lundby, C., 2013. Mitochondria express enhanced quality as well as quantity in association with aerobic fitness across recreationally active individuals up to elite athletes. Journal of Applied Physiology 114: 344-350.

Kuznetsova, A.V., Schneeberger, S., Seiler, R., Brandacher, G., Mark, W., Steurer, W., Saks, V., Usson, Y., Margreiter, R. and Gnaiger, E., 2004. Mitochondrial defects and heterogeneous cytochrome c release after cardiac cold ischemia and reperfusion. American Journal on Physiology - Heart and Circular Physiology 286: H1633-H1641.

LeBlanc, P.J., Peters, S.J., Tunstall, R.J., Cameron-Smith, D. and Heigenhauser, G.J., 2004. Effects of aerobic training on pyruvate dehydrogenase and pyruvate dehydrogenase kinase in human skeletal muscle. Journal of Physiology 557: 559-570.

Lemieux, H., Semsroth, S., Antretter, H., Höfer, D. and Gnaiger, E., 2011. Mitochondrial respiratory control and early defects of oxidative phosphorylation in the failing human heart. International Journal of Biochemistry and Cell Biology 43: 1729-1738.

Lopez-Rivero, J.L., Agüera, E., Monterde, J.G., Vivo, J. and RodríguezBarbudo, M.V., 1992. Skeletal muscle fiber size in untrained and endurance-trained horses. American Journal of Veterinary Research 53: 847-850.

Lopez-Rivero, J.L., Morales-Lopez, J.L., Galisteo, A.M. and Aguera, E., 1991. Muscle fibre type composition in untrained and endurancetrained Andalusian and Arab horses. Equine Veterinary Journal 23: 91-93.
Ohmura, H., Matsui, A., Hada, T. and Jones, J.H., 2013. Physiological responses of young thoroughbred horses to intermittent highintensity treadmill training. Acta Veterinaria Scandinavica 17: 55-59.

Pesta, D. and Gnaiger, E., 2012. High-resolution respirometry: OXPHOS protocols for human cells and permeabilized fibers from small biopsies of human muscle. Methods in Molecular Biology 810: 25-58.

Phielix, E., Schrauwen-Hinderling, V.B. and Mensink, M., 2008. Lower intrinsic ADP-stimulated mitochondrial respiration underlies in vivo mitochondrial dysfunction in muscle of male type 2 diabetic patients. Diabetes 57: 2943-2949.

Renner, K., Amberger, A., Konwalinka, G., Kofler, R. and Gnaiger, E., 2003. Changes of mitochondrial respiration, mitochondrial content and cell size after induction of apoptosis in leukemia cells. Biochimica and Biophysica Acta 1642: 115-123.

Ringmark, S., Lindholm, A., Hedenström U., Lindinger, M., Dahlborn, K., Kvart, C. and Jansson, A., 2015. Reduced high intensity training distance had no effect on VLa4 but attenuated heart rate response in 2-3-year-old Standardbred horses. Acta Veterinaria Scandinavica 57: $17-30$.

Rivero, J.L.L., Ruz, M.C., Serrano, A.L. and Diz, A.M., 1995. Effects of a 3 month endurance training programme on skeletal muscle histochemistry in Andalusian Arabian and Anglo-Arabian horses. Equine Veterinary Journal 27: 51-59.

Rivero, J.L., Ruz, A., Marti-Korff, S., Estepa, J.C., Aguilera-Tejero, E., Werkman, J., Sobotta, M. and Lindner, A., 2007. Effects of intensity and duration of exercise on muscular responses to training of thoroughbred racehorses. Journal of Applied Physiology 102: 1871-1882.

Serrano, A.L., Quiroz-Rothe, E. and Rivero, J.L., 2000. Early and longterm changes of equine skeletal muscle in response to endurance training and detraining. Pflugers Archive 441: 263-274.

Serteyn, D., De la Rebière de Pouyade, G., Sandersen, C., Salciccia, A., Grulke, S., Mouithys-Mickalad, A., Franck, T., Lejeune, J.P. and Ceusters, J., 2014. Muscle mitochondrial dysfunction in horses affected by acute laminitis. Bioenergetics 3: 120-124.

Votion, D.M., Fraipont, A., Goachet, A.G., Robert, C., Van Erck, E., Amory, H., Ceusters, J., De la Rebière de Pouyade, G., Franck, T., Mouithys-Mickalad, A., Niesten, A. and Serteyn, D., 2010. Alterations in mitochondrial respiratory function in response to endurance training and endurance racing. Equine Veterinary Journal Suppl. 38: 268-274.

Votion, D.M., Gnaiger, E., Lemieux, H., Mouithys-Mickalad, A. and Serteyn, D., 2012. Physical fitness and mitochondrial respiratory capacity in horse skeletal muscle. PloS One 7: e34890.

White, S.H., Warren, L.K., Li, C. and Wohlgemuth, S., 2015. Mitochondrial adaptations to submaximal exercise training in the gluteus medius and triceps brachii of young equine athletes. Journal of Equine Veterinary Science 35: 395. 
